\title{
An Approach to Continuous Pervasive Care of Remote Patients Based on Priority Based Assignment of Nurse
}

\author{
Paramita Sarkar ${ }^{1}$ and Ditipriya Sinha ${ }^{2}$ \\ ${ }^{1}$ AKCSIT, Calcutta University, Kolkata, India \\ mailtoparo@gmail.com \\ ${ }^{2}$ Megnadh Saha Institute of Technology, Kolkata, India \\ ditipriyasinha87@gmail.com
}

\begin{abstract}
Remote care of patient is now becoming a subject of major concern in healthcare services. Proposed work describes a pervasive system to assist continuously the patients who are at remote place from the connected hospital using a priority based classification and assignment of nurses to the high risk patients. The challenge lies in storage and management of the vast amount of real-time data originating from heterogeneous sources under dynamic situations. This paper attempts to design a new system consisting of several modules for managing real-time heterogeneous data. The overwhelming data could cause difficulty to decide over numerous patients to whom the care should be given first and then onwards. To solve this, the proposed system attempts to derive fuzzy rules to make decision based on priority among selected groups in a dynamic environment. This proposed model formulates an indexed hash key for high risk patients and proper nurse relatively.
\end{abstract}

Keywords: pervasive, context data, fuzzy rules, dynamicity, wearable sensor, hash key.

\section{Introduction}

Pervasive Computing is nowadays a growing research topic to improve the quality of remote healthcare service. It integrates seamlessly different heterogeneous technologies without user's awareness and intervention for the sake of user's benefit. Emerging ubiquitous or pervasive computing technologies offer 'anytime, anywhere, anyone' computing by decoupling users from devices. Context information used in pervasive environment provides adequate information regarding the changes occurred in any characteristics of users, place, and any event. This paper involves remote patient monitoring integrating body sensor network devices [1,2] that can upload a patient's real time contextual physiological information through a wrist worn wearable sensor device and a personal Smartphone for triggering risk alarm and dissemination to professional caregivers. Dynamic context are handled here by measuring automatic changes during a prolonged time and by mapping them into a predefined group. Some context data changes every second, and some every hour due to their dynamic nature. Recent advancement of pervasive computing has led increased accessibility to 
healthcare providers, centralized database storage, telemedicine services. Due to the growth in the number of chronically ill and weak people, it is becoming more difficult to take care of the patients in a central place. Therefore continuous monitoring of a remote patient in a pervasive way avoids the presence of a number of medical staff all-time at the patient's place. When the numbers of victims overwhelm the number of medical staff in a hospital in any emergency event, there are many challenges issues need to ration the medical staffs with proper assignment to patients [3]. Here this works attempts to search an appropriate nurse based on the computed hash key [section 4.3]. Continuous monitoring of patients from a remote hospital is highly desirable to take care of patients and providing suitable guidelines with proper medicine [4]. This paper includes heart rate variability as a new vital sign $[5,6]$ with other existing parameters. The design of proposed model inherits different existing real time systems for continuous monitoring [17], [19]. On the other hand, it develops a prioritised decision making using fuzzy inference and assignment of appropriate nurse to remote patient.

\section{Related Work}

In recent years lots of research, work and study in the related field have been carried out. A study of the review in this field revealed that several context aware pervasive system have been used regarding the services in emergency responses in rescue system, public healthcare system, other patient monitoring and alarm system, etc. This section is categorised into following subsection on the basis of real time implementation of pervasive computing.

\subsection{Priority Assignment}

Prioritized emergency operation plays an important role in remote healthcare services and evacuation system a lot. "Priority Assignment in Emergency Response" [3] is one of the application of priority assignment in pervasive environment. It formulated the priority assignment problem for each patient in triage system where each patient is evacuated from the mass casualty site to the hospital according to the priority set to them. This paper analysed the state dependent partial optimized policy and proposed a number of heuristic policies. Another "User-driven design of a context-aware application: an ambient-intelligent nurse call system" [8] focuses on the reasoning context data by the caregivers from various ambient sensors in the patient's room to answer a call from the patient. This paper describes the priority based of the proper nurse to the patient on the basis of the relation with patient.

\subsection{Knowledge Representation in Cloud Database}

It becomes crucial to store such huge and heterogeneous sensor real time data in a large database with proper representation and semantics of huge knowledge. Cloud computing is an obvious solution to access this large database from anyplace anytime in pervasive domain. "An approach for pervasive homecare environments focused 
on care of patients with dementia" [9] uses the ontology OWL [10] for homecare environment in pervasive system to represent the various relationships among domains and knowledge in the database implementing Semantic Web Rule Language (SWRL) and Semantic Query-Web Enhanced Language [11]. "Ontology for ContextAware Pervasive Computing Environments" [12] discussed the solution to the limitation of that problem using the architecture of the centralized pervasive context aware distributed system named as Context Broker architecture ( $\mathrm{CoBrA})$ which is a collection of different ontology (COBRA-ONT).

\subsection{Continuous Monitoring in Pervasive Environment}

For continuous monitoring of disable/older people and other patients in pervasive environment it is desirable to detect whether they have any risk factor or not. Several researches and designing approaches were performed to detect fall in recent few years $[13,14]$. "Evaluation of a threshold-based tri-axial accelerometer fall detection algorithm" [15] derives the threshold level for the fall detection. Different Body Sensors were developed for collecting and transmitting patient's physiological data for vital sign and activity recognition. "Human Activity Surveillance based on Wearable Body Sensor Network" [16] has proposed the advantages of a Body Sensor Network based architecture for wearable wireless monitoring system using an algorithm optimized for real time computing. To measure the impact of temperature as sleep deprivation in a human body "Accurate Temperature Measurements for Medical Research using Body Sensor Networks" [17] proposes a prototype of a body sensor network. Another vital sign of human body is heart rate/pulse rate. "Designing Heart Rate, Blood Pressure and Body Temperature Sensors for Mobile On-Call System" [18] paper describes the comparative study between different techniques and design issues for measuring heart rate, blood pressure and temperature. This paper focuses the different parameter used in measuring the vital signs of human body using ECG sensors and the Photoplethysmography (PPG). An unobtrusive method in "AMON: A Wearable Multiparameter Medical Monitoring and Alert System" [19] proposes the architecture and design of a miniature system for measuring heart rate using ECG, blood pressure, activity recognition and $\mathrm{SpO} 2$ for continuous monitoring high risk of cardiac and respiration patients. This system is optimized to collect vital signs of human body and evaluate these parameters continuously for communication through a gateway to the external database.

\section{Scope of the Work}

To implement pervasive environment in the system, heterogeneous data are needed to be stored in remote server. The state of the art study leads to the observation that not much attention has been paid to any mechanism to maintain heterogeneous real time dynamic data in the database. This proposed protocol describes a pervasive system to assist continuously the patients who are at remote place from the connected hospital using a priority based classification and assignment of nurses to the high risk patients. 


\section{$4 \quad$ Proposed Work}

The proposed work is to design a system on the pervasive care environment for remote patient. This system emphasizes on the allotment of a caregiver to the remote patients according to the condition of the patients.

\subsection{Assumption}

- The wearable device analyses and processes every sensor signal in such a way that after every 5 minutes it can send data to the patient's Smartphone.

- Threshold value has been determined for Heart Rate, Blood Pressure, Temperature, Accelerometer (for Activity Recognition, Fall Detection) and Heart Rate Variability $[5,6]$ and $[15,16,17,18,19]$.

- The continuous monitoring is attempted as long as the patient's wearable 4-array sensor wrist band is connected with server through the Bluetooth.

- Only the dynamic context data are considered for classification in every $30 \mathrm{mi}-$ nutes.

- The priority of a patient is classified into five groups Urgent, High, Medium, and Low, and Very Low depending on the severity of the context data coming from the Smartphone.

- Work Constraints: The following work constraints should not be violated during the allotment of the nurse to patients. They are as follows:

1. No nurses will have workload more than 8 hours in a whole day.

2. More experienced resources in a particular department should be allocated to high priority job.

3. Expertise of a resource in a particular department should be given preferred most.

4. Minimum number of working days in a week is 5 .

5. Minimum gap between two shifts is 12 hours.

6. Maximum number of consecutive days of duties in a week will be 3 .

- At the server site, the schedule of nurses for each department and for each day of a week stored in its database. The existing schedule of nurses is updated every 30 minutes inside the server to calculate the remaining duty hours and key value of a nurse.

- Weights are provided to represent for the level of importance for each constraint.

- The allotment of the nurses is prioritized according to the lower value of the key c.

- Two different priority queues are needed for prioritizing the patients and the nurses.

\subsection{Data Dictionary}

- $\mathrm{E}_{\mathrm{di}}=$ number of experience in years of $\mathrm{i}^{\text {th }}$ nurse of a particular department say $\mathrm{d}$

- $\mathrm{H}_{\mathrm{i}=}$ total duty hours of $\mathrm{i}^{\text {th }}$ nurse in a day.

- $\mathrm{D}_{\mathrm{i}}=$ highest qualification of $\mathrm{i}^{\text {th }}$ nurse in a particular domain related to the particular department 
- $\mathrm{nw}_{\mathrm{i}}=$ Minimum number of working day (w) of $\mathrm{i}^{\text {th }}$ in a week

- $\mathrm{ng}_{\mathrm{i}}=$ minimum gap $(\mathrm{g})$ between two successive shift of duty of $\mathrm{i}^{\text {th }}$ nurse.

- $\mathrm{xcd}_{\mathrm{i}}=$ Maximum number of consecutive duties (c) of $\mathrm{i}^{\text {th }}$ nurse in days of a week.

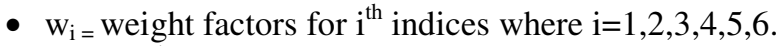

- $\mathrm{p} \_\mathrm{id} \mathrm{d}_{\mathrm{i}} \mathrm{i}^{\text {th }}$ patient's id given by the hospital

- threshold $_{n i}=$ corresponding threshold value of $\mathrm{n}^{\text {th }}$ feature and $\mathrm{i}^{\text {th }}$ patient

- feature_value $e_{n i}=$ value of the $n^{\text {th }}$ context data of $i^{\text {th }}$ patient

- num_gr_f $f_{n i}=$ associating group numbers to which $n^{\text {th }}$ feature of $i^{\text {th }}$ patient belongs to.

- cnt_gr_f $f_{n i}=$ total number of groups to which $n^{\text {th }}$ feature of $i^{\text {th }}$ patient belongs to.

- feature_priority ${ }_{n i}=$ priority of $n^{\text {th }}$ feature of $i^{\text {th }}$ patient

- gr_id $_{\mathrm{i}}=$ group id of $\mathrm{i}^{\text {th }}$ feature

- $g r \_m_{i}=$ feature_name of $n^{\text {th }}$ patient in the $i^{\text {th }}$ gr_id

- $\quad$ __hr $r_{i}=$ Heart Rate of $i^{\text {th }}$ patient, $p \_b p_{i}=$ Blood Pressure of $i^{\text {th }}$ patient,p_acc $c_{i}=$ of accelerometer data $i^{\text {th }}$ patient, $p \_$temp $=$temperature of $i^{\text {th }}$ patient, $p \_$hrv ${ }_{i}=$ Heart Rate Variability of $i^{\text {th }}$ patient.

- p_arr_time ${ }_{\mathrm{i}}=$ arrival time of a sensor data of $\mathrm{i}^{\text {th }}$ patient

Table 1. Database Record Table

\begin{tabular}{|c|c|}
\hline Record & Field Name \\
\hline $\begin{array}{l}\text { Patient's } \\
\text { record }\end{array}$ & 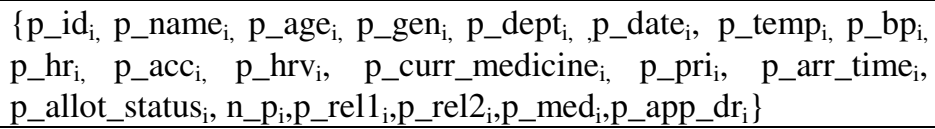 \\
\hline $\begin{array}{l}\text { Nurse's } \\
\text { Record }\end{array}$ & 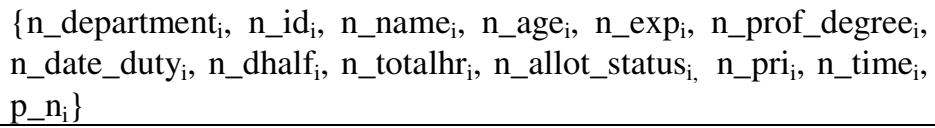 \\
\hline $\begin{array}{l}\text { Feature's } \\
\text { record }\end{array}$ & 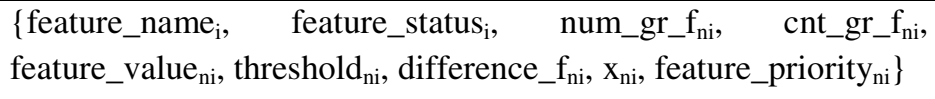 \\
\hline $\begin{array}{l}\text { Doctor's } \\
\text { record }\end{array}$ & $\left\{\mathrm{dr}_{-}\right.$department, $\mathrm{dr} \_$name $_{\mathrm{i}}, \mathrm{dr} \_$degree $_{\mathrm{i}}, \mathrm{dr} \_$time $_{\mathrm{i}}, \mathrm{dr} \_$date $\left._{\mathrm{i}}\right\}$ \\
\hline $\begin{array}{l}\text { Group } \\
\text { record }\end{array}$ & $\left\{\mathrm{gr}_{-} \mathrm{id}, \mathrm{gr}, \mathrm{gr} \_\mathrm{mem}_{\mathrm{i}}\right\}$ \\
\hline Key record & key $_{\mathrm{i}}$, hfnc $_{\mathrm{i}}$ \\
\hline
\end{tabular}

\subsection{Definition}

Definition 1. Indexed Key defines-the minimum key is defined to search the appropriate nurse from the database without avoiding all work constraints is defined as the total cost function:

$$
\mathrm{C}=\mathrm{E}_{\mathrm{di}} \times \mathrm{w}_{1}+\mathrm{H}_{\mathrm{i}} \times \mathrm{w}_{2}+\mathrm{D}_{\mathrm{i}} \times \mathrm{w}_{3}+\mathrm{nw}_{\mathrm{i}} \times \mathrm{w}_{4}+\mathrm{ng}_{\mathrm{i}} \times \mathrm{w}_{5}+\mathrm{xcd}_{\mathrm{i}} \times \mathrm{w}_{6}
$$

$\mathrm{w}_{\mathrm{i}}$ [Section 4.2] is in the range from 1 to 6 according to the value of six indices, such that if a nurse has highest experience in a particular department $\left(\mathrm{E}_{\mathrm{di}}\right)$, the weight 
factor $\mathrm{w}_{\mathrm{i}}$ for that constraint will be 1 . This minimum cost function will be treated as the key for index in the hash function.

Definition 2. The hash function is required to search proper nurse_id from the hash table. A hash function of key is

$$
\text { hash(key) }=\text { key } \% \text { hash table size }
$$

where key is defined as total cost function C. Searching will be performed based on the indexed key such that the hash function of that key would probably returns the location of the key value of the corresponding nurse.

Definition 3.The difference_ $f_{n i}$ is defined as the difference between the threshold of a particular feature $n$ of $i^{\text {th }}$ patient.

$$
\text { difference_f }_{\text {ni }}=\mid\left(\text { threshold }_{\text {ni }}-\text { feature_value }_{n i}\right) \mid
$$

This value will be computed in the computation module inside the server at the time of arrival of every context data.

\subsection{Proposed Pervasive Remote Patient Care with Proper Nurse Assignment Model}

In order to assist this work, a proposed model of the system's architecture is implemented at the server of the particular hospital. The server communicates with the Smartphone of the patient and the nurse through GSM. The proposed model can finally generate an assignment of appropriate nurses to high risk remote patients on the basis of the proposed priority inside the classification model. Figure 1 shows architecture of the proposed model inside the server. The proposed system has four connected parts labelled here as COMPUTATION MODULE, DATA MODULE, CLASSIFICATION MODULE,SCHEDULING MODULE, and UPDATATION MODULE.

Table 2. List of Abbreviation used in the Figure 1

\begin{tabular}{|l|l|l|}
\hline No. & Abbreviation & Meaning \\
\hline 1 & R_p,n,k & $\begin{array}{l}\text { Record of patients, nurses and index } \\
\text { key }\end{array}$ \\
\hline 2 & R_p,n, & Record of patients and nurses \\
\hline 3 & R_p & Record of patients \\
\hline 4 & R_p,n,k,f,g & $\begin{array}{l}\text { Record of patients, nurses, features and } \\
\text { group }\end{array}$ \\
\hline 5 & s_data & $\begin{array}{l}\text { Sensor data coming from wearable } \\
\text { device }\end{array}$ \\
\hline 6 & MSG,con & Message and computed context data \\
\hline 7 & N_MSG & Notification message \\
\hline
\end{tabular}




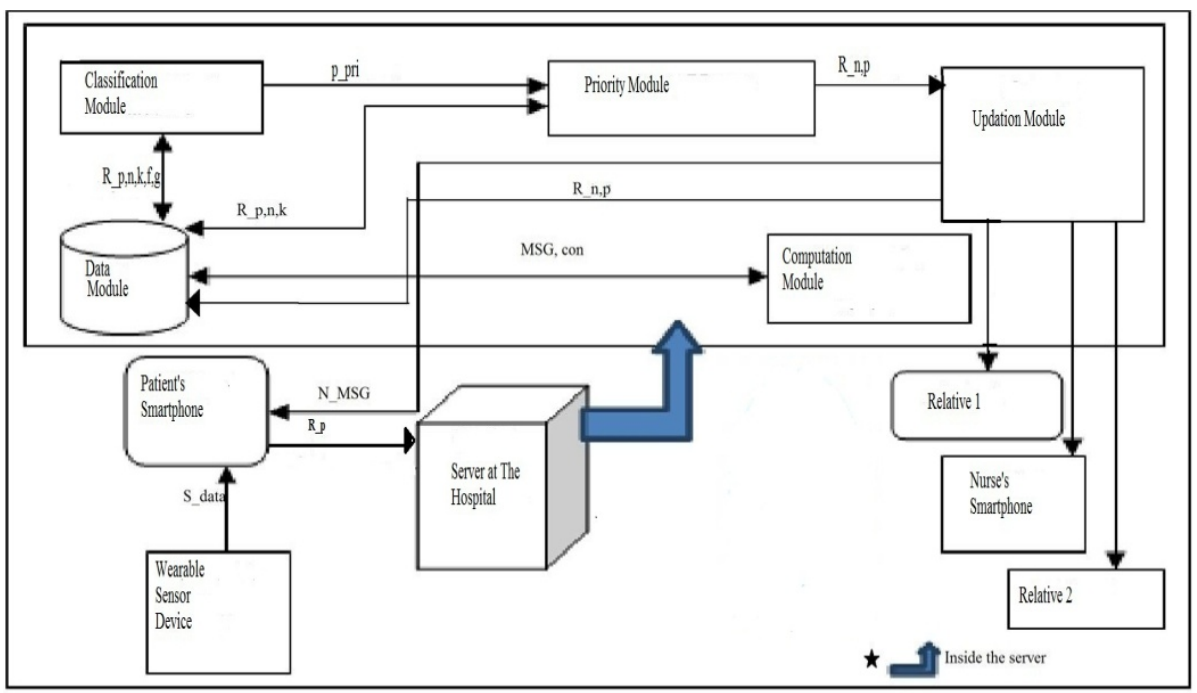

Fig. 1. Architecture of the Proposed Pervasive care Environment of remote patients

\subsubsection{Module I: Computation Module}

Data are coming continuously from the patient's wearable device to the Smartphone via Bluetooth.

1. Patient's records [Section 4.2] are sent from patient's Smartphone to the hospital server. Smartphone can send this updated record of the corresponding patient every 5 minutes until any notification received from the hospital.

2. Option is created for new patient to be registered to the connected hospital whenever he/she wears the device first time as follows.

3. Registration: On the particular page of the website of the hospital, responsive staff will check for registration of the patient wearing the sensor/

a) if $\mathrm{p} \_\mathrm{id}_{\mathrm{i}}==\mathrm{NULL}$

then, Set status: = "Not registered" and server sends REQ_MSG to the patient's Smartphone with Accept or Ignore option.

b) If the patient accepts this message then, server asks for confirmation of sending patient id to the new patient

c) After confirming that message, new patient will receive the new $\mathrm{p}_{-} \mathrm{id}_{\mathrm{i}}$ and $\mathrm{p} \_\mathrm{dept}_{\mathrm{i}}$. Patient's records are received from the Smartphone by the server.

d) At this time two phone numbers of patient's most close relatives/friends are required for further notification.

4. Existing $\mathrm{i}^{\text {th }}$ patient's record [Section 4.2] is sent from the Smartphone at regular time interval to the server.

5. At the hospital terminal, those data are automatically compared to the threshold $_{\text {ni }}[$ Section 4.2] for different context data.

if difference_ $f_{n i} \neq 0$ [Equation (3)] 
then, difference_ $f_{\text {ni }}$ will be inserted in the patient's record [Section 4.2] and will be updated in the data module. Otherwise, the context data of $i^{\text {th }}$ patient will be discarded. Thus the Computation module reduces the size of the database. Finally it sends the updated records to the classification module for further processing.

\subsubsection{Module II: Data Module}

Inside the server there will be separate database to store records of all patients, nurses, doctors, context features, their group, nurse's schedule and for key with hash function for every nurse. A database of fuzzy inference mechanism is attached in this Data Module.

- Fuzzy rules are stored in the separate database of this module. From this database classification module will be able to infer the priority of the patient.

- For this architecture there is also a record of five contextual health data [Section 4.2]. These are the most vital sign to decide the health risk of a patient.

\subsubsection{Module III: Classification Module}

Phase 1: Checking Dynamicity of Real-Time Contextual Data. In every 5 minutes patients' record at the hospital will be updated by the coming data.

1. Initialize time duration $\mathrm{t}_{0}:=0 \mathrm{sec}$

2. Repeat the step 4 while $\mathrm{t}_{\mathrm{i}}<1800 \mathrm{sec}$

3. [ check changes frequently in 30 minutes duration] if Idifference_ $f_{n i} \mid$ at $t_{i+1} \neq \mid$ difference_ $f_{n i} \mid$ at $t_{i}$ [Equation (3)] then Set feature_status $\mathrm{i}_{\mathrm{i}}=$ "dynamic"

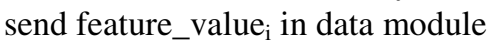

4. Else save the old feature_value

Fuzzy Sets for "priority". In the proposed system, "priority" is defined as a linguistic variable to $\mathrm{x}$. Linguistic variable are termed as $\mathrm{x}, \mathrm{F}(\mathrm{x}), \mathrm{U}$ and $\mathrm{M}$. $\mathrm{F}(\mathrm{x})$ is termed as the set of $\mathrm{x}$, the set of names or linguistic values assigned to $\mathrm{x}$, with each value is a fuzzy variable defined in $\mathrm{U} . \mathrm{M}$ is membership where semantic rules are associate with each variable [20]. $\mathrm{T}$ (priority) $=\{$ low, very low, high, medium, urgent $\} . U=\{-\alpha,+\alpha\} . \mathrm{M}$ defines the membership function of each fuzzy variable for example, $M$ (very low)= fuzzy set priority below $20 \%$ with membership of $\mu_{\text {very low. The }}$ M (low) and M (high) are expressed as $\mu_{\text {low }}$ and $\mu_{\text {high }}$ respectively.

$$
\begin{aligned}
\mu_{\text {low }}(x) & =1 /[1+(x / 20)]^{4} \\
\mu_{\text {high }}(x) & =1 /\left[1+((x-(+\alpha)) / 20)^{6}\right]
\end{aligned}
$$

Concentration and Dilation of fuzzy membership function. $\mathrm{M}(\mathrm{Urgent})=\mu_{\text {urgent }}(x)$.

Not low not high means $\mathrm{M}($ medium $)=\mu_{\text {medium }}(x)$. Low but not too low means $\mathrm{M}($ medium $)=\mu_{\text {medium }}(x)$. 


$$
\mu_{\text {urgent }}(x)=\left\{1 /\left[1+((x-(+\alpha)) / 20)^{6}\right]\right\}^{0.5}
$$

$\mu_{\text {medium }}(x)=\min \left\{\left(\frac{1}{1-1 /\left[1+(\mathrm{x} / 20)^{4}\right]}\right) ;\left(1-\frac{1}{\left[1+((\mathrm{x}-(+\alpha)) / 20)^{6}\right]}\right)\right\}$

$\mu_{\text {medium }}(x)=\min \left\{\frac{1}{\left[1+(\mathrm{x} / 20)^{4}\right]} ;\left(\left(\frac{1}{\left[1+((\mathrm{x}-(+\alpha)) / 20)^{6}\right]}\right)^{0.5}\right)\right\}$

$\mu_{\text {very low }}(x)=\min \left\{1 /[1+(x / 20)]^{4} ;\left(\left(1 /\left[1+((x-(+\alpha)) / 20)^{6}\right]\right)^{0.5}\right)\right\}$

Phase 2: Priority Based Classification Algorithm. Group Assignment of specific feature in one or more than one group of clinically related context data has been done. At this phase a priority based classification algorithm is derived using Fuzzy memebership is computed according to the different range and that are assigned to each patient.

1. Compute the difference between the received feature_value ${ }_{n i}$ and the threshold ${ }_{n i}$ [Section 4.2] in percentage and that will be stored.

2. Set $x_{n i}:=\mid\left(\right.$ difference_f $\left.f_{n i} / 100\right) \mid[$ Equation (3) ]

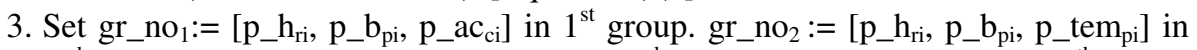
$2^{\text {nd }}$ group. gr_no $3:=\left[\mathrm{p} \_\mathrm{ac}_{\mathrm{ci}}, \mathrm{p} \_\right.$tem $\left.\mathrm{pi}_{\mathrm{i}}\right]$ in $3^{\text {rd }}$ group. gr_no $\mathrm{gr}_{4}:=\left[\mathrm{p} \_\mathrm{hr}_{\mathrm{vi}}\right]$ in $4^{\text {th }}$ group [Section 4.1]

4. if (feature_name $!_{i}=$ NULL) then

a) search its group members using num_gr_f $f_{i}$ and cnt_gr_f $f_{n i}$ [Section 4.2]

b) [Initialize counter count for checking in all groups] Set c:=1

c) Repeat the following step while $\mathrm{c}<=$ cnt_gr_f $\mathrm{f}_{\mathrm{ni}}$ for $\mathrm{n}^{\text {th }}$ feature in $\mathrm{i}^{\text {th }}$ group.

a) Make decision for priority assignment according to the following Decision Tree based on the value of $\mathrm{x}_{\mathrm{ni}}$.

b) if $|20|<=x_{n i}<=|80|$ of feature_value ${ }_{n i}$ then, Set feature_priority automatically according to the following decision.

5. Repeat the following steps until the final priority assignment is completed and the communication link is active. Let $\mathrm{x}_{\mathrm{i}}$ is any sensor data of $\mathrm{i}^{\text {th }}$ patient

6. if $\mathrm{M}\left[\mathrm{x}_{\mathrm{ni}}\right]=\mu_{\text {urgent }}(x)$ [Equation (6)] then, Set feature_priority $\mathrm{y}_{\mathrm{i}}$ : = "urgent" and p_pri $i_{i}:=5$ Else

7. if $\mathrm{M}\left[\mathrm{x}_{\mathrm{ni}}\right]=\mu_{h i g h}(x)$ [Equation (5)] then Set feature_priority $\mathrm{i}_{\mathrm{i}}$ : = "High" and p_pri $i_{i}:=4$ and if ( num_gr_f $\left.f_{n} !=N U L L\right)$ then

a) if all feature_values ${ }_{n i}$ of $g_{-} \_m_{i}$ have $x_{n i}$ is in the range $\{|50 \%-80 \%|\}$ then, Set feature_priority $\mathrm{i}_{\mathrm{i}}=$ urgent $\mid$ high $\mid$ mediuml low according to the relative group

b) Else Set feature_priority $\mathrm{i}_{\mathrm{i}}=$ high $\mid$ medium | low according to the relative group

8. Else Set feature_priority ${ }_{\mathrm{i}}:=$ "High" and p_pri $\mathrm{i}_{\mathrm{i}}:=4$

9. Repeat the above steps for all group and for all ranges according to the following decision structure. 


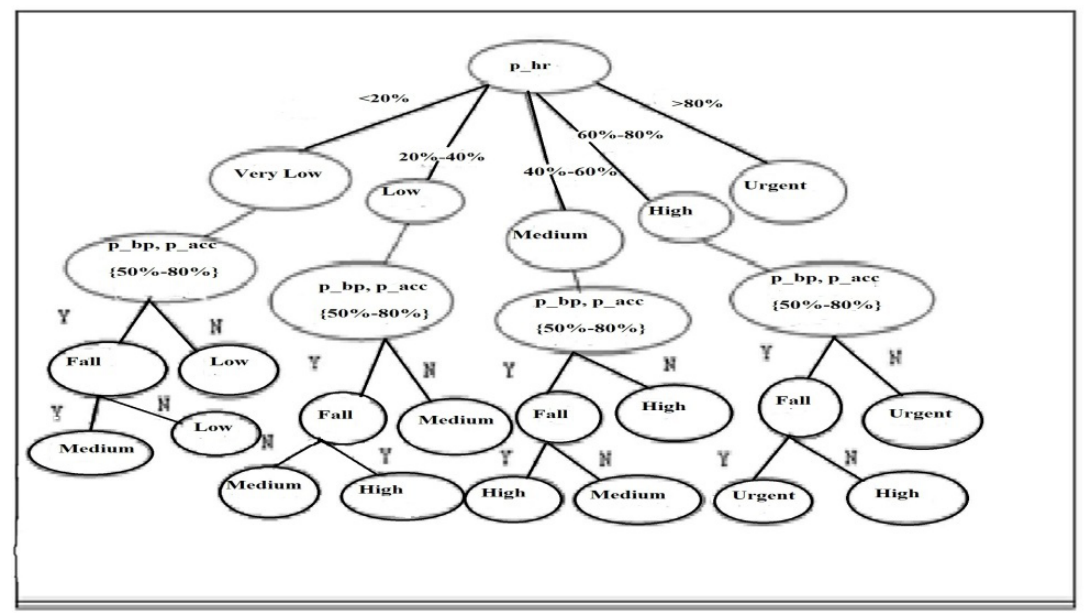

Fig. 2. Decision Tree architecture for priority assignment for group2 with Heart rate, Blood pressure, and temperature

\subsubsection{Module IV: Priority Module}

This module receives the list of all critical patients that have rapid variation in their physiological context information compared to the threshold value.

1. if $\left(p_{-}\right.$pri $\left._{i}==5\right)$ then $p_{-}$name ${ }_{i}$ are brought to the hospital immediately. The details information of that patient is available with all in his/her record [Section 4.2] in the data module.

2. Else Higher $\mathrm{p}_{-} \mathrm{id}_{\mathrm{i}}$ takes place at the front of the priority queue and deleted from it [Section 4.1] according the higher priority.

3. if ( $\left(p \_p r i i_{i}==p \_p r i_{i+1}\right)$ then if (p_arr_time $e_{i}<$ p_arr_time $\left.{ }_{i+1}\right)$ then Set p_pri $i_{i}>$ p_pri $i_{i+1}$.

4. if $\left(\mathrm{n}_{-} \mathrm{dep}_{\mathrm{i}}==\mathrm{p} \_\mathrm{dep} \mathrm{p}_{\mathrm{i}}\right)$, then $\mathrm{key}_{\mathrm{i}}$ and hash function $\left(\mathrm{hfnc}_{\mathrm{i}}\right)$ [Section 4.2 and 4.3] of all nurses [Equation (1) and (2)] are stored.

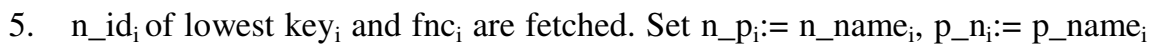
records are also updated.

6. Until the first allotment is complete, the next patient should have to wait in the priority queue.

7. This new record is sent to the next module of the architecture.

\subsubsection{Module V: Updation Module}

The updation module performs like the informer from the server to the users.

1. After the allotment of the available nurses, Set p_alott_status $\mathrm{s}_{\mathrm{i}}$ : = 'yes' and n_alott_status $:$ : = 'yes' 
2. The patient will receive a NOTI_MSG_p from the remote server about the allotment, and updated $n \_i d_{i}, n_{-}$time $e_{i}$. At the same time, the corresponding nurse will also receive the same alert message N_MSG with updated p_id and $\mathrm{p} \_\mathrm{add}_{\mathrm{i}}$ and updated schedule record.

3. If no available nurse is found then Set p_alott_status $\mathrm{i}_{\mathrm{i}}$ : = "wait". It sends a list of prescribed p_curr_medicine ${ }_{i}$ and $a p p \_d r_{i}[$ Section 4.2] to patient's Smartphone.

4. N_MSG [Section 4.4.1] are also sent to relatives' smartphone with the same updating for providing better support of this mechanism.

\section{Conclusion}

This paper designs the pervasive system to alert the healthcare providers for allotting a suitable nurse to the high risk patients. Some vital signs have been used as contextual sensor data which are continuously gathered and updated at the remote server at a regular interval. The focus of this work is to meet the challenges of existing work in the related field. A priority based classification using fuzzy logic is devised for alerting the health risk of the registered patients of a particular hospital. This work needs to be compared with other prioritized nurse assignment protocol in future so that this work can be validated. A scheduling plan will be generated in dynamic real time environment as well as a proper knowledge representation mechanism will be implemented using Semantic Web Rule Language (SWRL) [10] and Semantic Query-Web Enhanced Language [11] for generating updated knowledge and rules in huge database. Intelligent and a faster method for group assignment of the context data in the server will also a challenging task for future work.

\section{References}

1. Ullah, S., Higgin, H., Siddiqui, M.A., Kwak, K.S.: A Study of Implanted and Wearable Body Sensor Networks. In: Nguyen, N.T., Jo, G.-S., Howlett, R.J., Jain, L.C. (eds.) KES-AMSTA 2008. LNCS (LNAI), vol. 4953, pp. 464-473. Springer, Heidelberg (2008)

2. Lo, L.P.B., Thiemjarus, S., King, R., Yang, G.Z.: Body sensor network - A wireless sensor platform for pervasive healthcare monitoring. In: Adjunct Proceedings of the 3rd International Conference on Pervasive Computing (PERVASIVE 2005), pp.77-80 (May 2005)

3. Jacobson, U.E., Argon, T.N., Ziya, S.: Priority Assignment in Emergency Response. Journal Operations Research. ACM Digital Library 60(4), 07-08, 813-832 (2012)

4. Mirkovic, J., Norway, B.H., Rulland, C.M.: Framework for the development of ubiquitous patient support systems. In: 6th International Conference on Pervasive Computing Technology for Health Care, May 21-24. IEEEXPLORE (2012)

5. Malik, M.: Heart Rate Variability, Standards of Measurement, Physiological Interpretation, and Clinical Use. AHA Journals, Circulation 93, 1043-1065 (1996), doi:10.1161/01.CIR.93.5.1043 
6. Norton, C.L., Fair, M.J., Taylor-Piliae, E.R., Mahbouba, H.M., Iribarren, C., Fortmann, P.S.: Cardiovascular Risk Factors and Heart Rate Variability in an Older Cohort. Abstract 2096 114:II_425 (2006)

7. Kung, H.-Y., Lin, M.-H., Hsu, C.-Y., Liu, C.-N.: Context-Aware Emergency Remedy System Based on Pervasive Computing. In: Yang, L.T., Amamiya, M., Liu, Z., Guo, M., Rammig, F.J. (eds.) EUC 2005. LNCS, vol. 3824, pp. 775-784. Springer, Heidelberg (2005)

8. Ongenae, F., Duysburght, P., Verstraete, M., Sulman, N., Bleumerst, L., Jacabst, A., Ackaert, A., Zutter, D.S., Verstichel, F., Turck, D.: User-driven design of a context-aware application: an ambient-intelligent nurse call system. In: 6th IEEE - International Conference on Pervasive Computing Technologies for Healthcare, pp. 205-210 (2012)

9. Bastiania, E., Librelottoa, R.G., Freitasa, O.L., Pereirab, R., Brasilc, B.M.: An approach for pervasive homecare environments focused on care of patients with dementia. In: Bastiani, E., et al. (eds.) Procedia Technology, vol. 9, pp. 921-929. Elsevier (2013), doi:10.1016/j.protcy.2013.12.103

10. Bechhofer, S., van Harmele, F., Hendler, J., Horrocks, I., McGuinness, L.D., PatelSchneid, F., Stein, A.: Owl web ontology language reference. W3C Recommendation (2004), http: / / www.w3 . org/TR/owl-ref /

11. Horrocks, I., Patel-Schneider, F.P., Boley, H., Tabet, S., Grosof, B., Dean, M.: SWRL: a semantic web rule language combining OWL and ruleML (2004), W3C Member Submission, http: / /www.w3 .org/Submission/SWRL/

12. Chen, H., Finin, T., Joshi, A.: An Ontology for Context-Aware Pervasive Computing Environments. supported by DARPA contract F30602-97-1-0215, NSF award 9875433, NSF award 0209001, and Hewlett Packard (2003)

13. Diaz, A., Prado, M., Roa, L., Reina-Tosina, J., Sa'nchez, G.: Preliminary evaluation of a full-time falling monitor for the elderly. In: Proceedings of the 26th Annual International Conference of the IEEE-EMBS, pp. 2180-2183 (2004)

14. Hwang, J.Y., Kang, J.M., Jang, Y.W., Kim, H.C.: Development of Novel algorithm and real-time monitoring ambulatory system using Bluetooth module for fall detection in the elderly. In: Proceedings of the 26th annual international conference of the IEEE-EMBS (2004)

15. Bourke, K.A., O’Brien, V.J., Lyons, M.G.: Evaluation of a threshold-based tri-axial accelerometer fall detection algorithm. Elsevier, ScienceDirect, Gait \& Posture 26, 194-199 (2007)

16. Kantoch, E., Augustyniak, P.: Human Activity Surveillance based on Wearable Body Sensor Network. In: AGH University of Science and Technology, Kraków, Poland, Computing in Cardiology, vol. 39, pp. 325-328 (2012)

17. Boano, C.A., Lasagni, M., Römer, K., Lange, T.: Accurate Temperature Measurements for Medical Research using Body Sensor Networks. In: 14th IEEE International Symposium on Object/Component/Service-Oriented Real-Time Distributed Computing Workshops, Newport Beach, CA, pp. 189-198 (2011)

18. Designing Heart Rate, Blood Pressure and Body Temperature Sensors for Mobile On-Call System (2010), http: / / digitalcommons.mcmaster.ca/ee4bi6/39/

19. Anliker, U., Ward, J.A., Lukowicz, P., Tröster, G., Dolveck, F., Baer, M., Keita, F., Schenker, E., Catarsi, F., Coluccini, L., Belardinelli, A., Shklarski, D., Alon, M., Hirt, E., Schmid, R., Vuskovic, M.: AMON: A Wearable Multiparameter Medical Monitoring and Alert System. IEEE Transaction on Information Technology in Biomedicine 8(4), 415-427 (2005)

20. Artificial Intelligence Application in Construction, http: / /osp.mans.edu.eg/elbeltagi 